\title{
Electronic structure and optical properties of metallic nanoshells
}

\author{
P. Nordlander and E. Prodan \\ Department of Physics and Rice Quantum Institute, M.S. 61, Rice University \\ Houston, TX 77251-1892, USA
}

\begin{abstract}
The electronic structure and optical properties of metallic nanoshells are investigated using a jellium model and the Time Dependent Local Density Approximation (TDLDA). An efficient numerical implementation enables applications to nanoshells of realistic size with up to a million electrons. We demonstrate how a frequency dependent background polarizability of the jellium shell can be included in the TDLDA formalism. The energies of the plasmon resonances are calculated for nanoshells of different sizes and with different dielectric cores, dielectric embedding media, and dielectric shell backgrounds. The plasmon energies are found to be in good agreement with the results from classical Mie scattering theory using a Drude dielectric function. A comparison with experimental data shows excellent agreement between theory and the measured frequency dependent absorption spectra.
\end{abstract}

Keywords: Nanoshells, nanophotonics, nanooptics, nanoparticles, TDLDA

\section{INTRODUCTION}

The optical properties of metallic nanoparticles are of considerable fundamental and technological interest. ${ }^{1} \mathrm{~A}$ particularly interesting nanoparticle is the metallic nanoshell, ${ }^{2}$ which consists of a metallic layer grown over a solid dielectric core. The plasmon frequency, which determines the nanoshell optical properties, can be tuned over a wide spectral range by simply varying the ratio of inner to outer diameter of the shell. The tunability of the optical properties of metallic nanoshells enables several important applications such as resonant photooxidation inhibitors, ${ }^{3}$ optical triggers for drug delivery implants, ${ }^{4,5}$ environmental sensors, ${ }^{6}$ and Raman sensors. ${ }^{7}$

Although classical Mie scattering seems to account well for the optical properties of metallic nanoshells, there is clearly need for a more microscopic description. A microscopic understanding of the electronic properties of nanoshells is a prerequisite for a systematic and rational modification of their electronic and optical properties. We have recently started the development of a fully quantum mechanical model for the electronic properties of metallic nanoshell using density functional theory. ${ }^{8-13}$ In this paper we will review the general features of our approach and present some new results. The emphasis will be on the effects of dielectric backgrounds on the electronic structure and optical properties of metallic nanoshells.

\section{ELECTRONIC STRUCTURE}

TDLDA is a well established method, ${ }^{14}$ which allows surprisingly accurate calculations of the electronic and optical properties of nanoparticles. The method was extensively applied to small metal clusters and proven to be in excellent agreement with more exact calculations and experiment. ${ }^{15,16}$ The method was recently implemented to shell geometries and proven to be in good agreement with the existent classical and semi-classical approaches. ${ }^{10-13}$ TDLDA involves two steps. The first step consists of self-consistent calculation of the electronic structure and the independent electron response functions of the nanoparticle. The second step consists of solving self-consistently the RPA equation for the screened response function of the system. Our implementation of the first step to shell geometries has been discussed in details previously, ${ }^{9-11}$ and therefore only the main points will be presented here.

Further author information: (Send correspondence to P.N.)

P.N.: E-mail: nordland@rice.edu, Telephone: 17133485171

E.P.: E-mail: emprodan@rice.edu, Telephone: 17133483541 


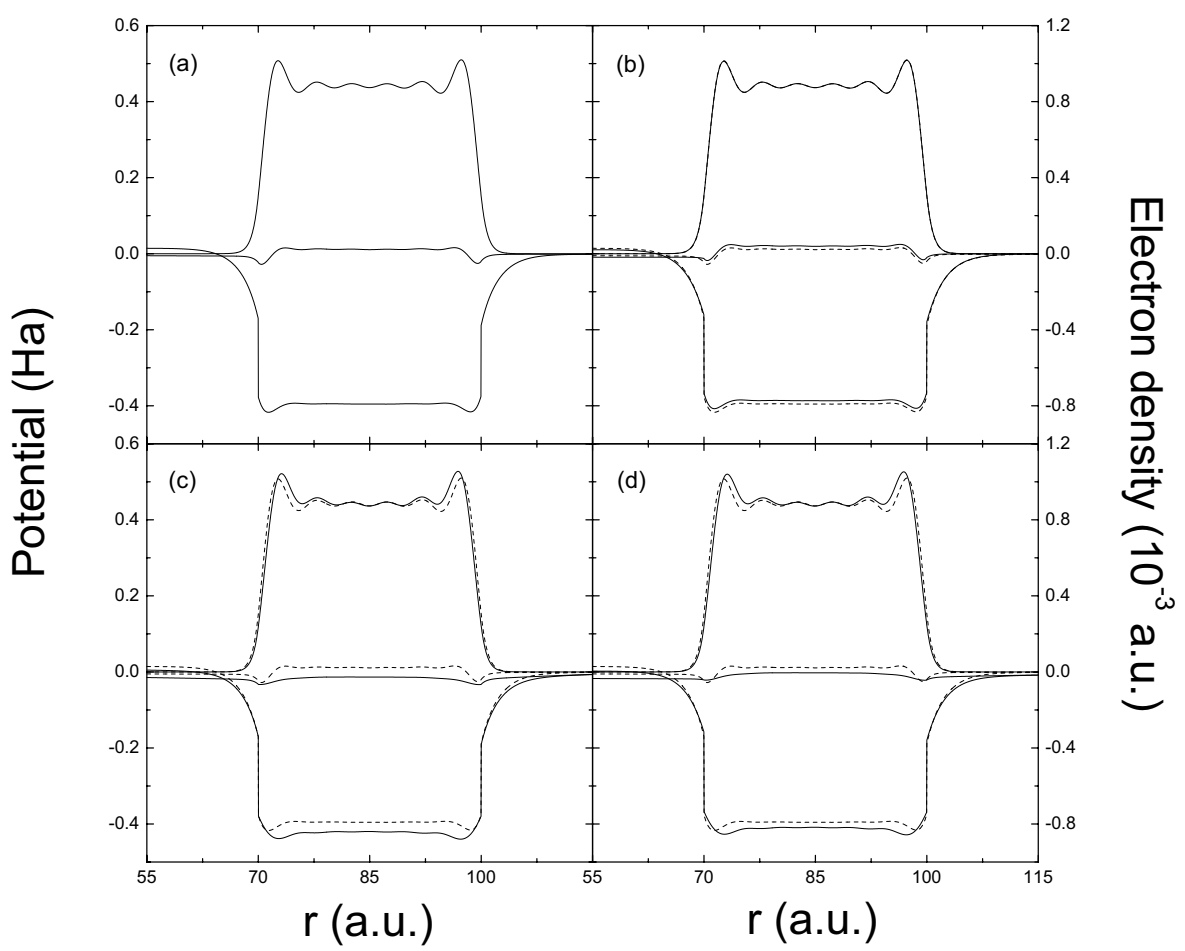

Figure 1. The effect of different dielectrics on the electronic structure of a $(70,100)$ a.u. gold nanoshell. The curves in each panel represent, from the bottom and up, the effective potential, the Coulomb potential (the values are on the left axis) and the electron density (the values on the right axis). Different panels correspond to: a) $\varepsilon_{C}=1, \varepsilon_{E}=1$ and $\varepsilon_{J}=1$; b) $\varepsilon_{C}=5, \varepsilon_{E}=2$ and $\varepsilon_{J}=1$; c) $\varepsilon_{C}=1, \varepsilon_{E}=1$ and $\varepsilon_{J}=8$; d) $\varepsilon_{C}=5, \varepsilon_{E}=2$ and $\varepsilon_{J}=8$. For comparison, in panels b)-d) we have included with dashed lines the electronic structure when no dielectrics are present, i.e. $\varepsilon_{C}=\varepsilon_{E}=\varepsilon_{J}=1$.

The electronic structure of the metallic nanoshells is determined from the Kohn-Sham equations

$$
\left(-\frac{1}{2} \Delta+V_{e x t}+v_{H}+v_{x c}[n]\right) \phi_{i}=\varepsilon_{i} \phi_{i}
$$

where

$$
n(\vec{x})=\sum_{i}\left(1+e^{\beta\left(\varepsilon_{i}-\mu\right)}\right)^{-1}\left|\phi_{i}(\vec{x})\right|^{2} .
$$

The chemical potential is fixed by the condition:

$$
N=\sum_{i}\left(1+e^{\beta\left(\varepsilon_{i}-\mu\right)}\right)^{-1}
$$

$N$ being the number of conduction electrons. The term $V_{\text {ext }}$ represents a uniform background potential inside the shell. Its value is fixed such that the converged calculations lead to a ionization potential equal to $5.4 \mathrm{eV}$, appropriate for gold. To avoid problems associated with the large degeneracies in spherically symmetric systems, we perform the calculations at low but finite temperature. The self-consistency is achieved by employing the usual iterative method. In the jellium approximation, the system becomes spherically symmetric and consequently the Kohn-Sham orbitals must be of the form $r^{-1} u_{l k}(r) Y_{l m}(\hat{r})$. In this case the Kohn-Sham equations are reduced 
to a set of coupled radial Schroedinger equations:

$$
\left(-\frac{1}{2} \frac{d^{2}}{d r^{2}}+\frac{l(l+1)}{2 r^{2}}+v_{e f f}[n]\right) u_{l k}(r)=\varepsilon_{l k} u_{l k}(r),
$$

where

$$
n(r)=\frac{2 s+1}{r^{2}} \sum_{l, k} \frac{2 l+1}{1+e^{\beta\left(\varepsilon_{l k}-\mu\right)}}\left|u_{l k}(r)\right|^{2} .
$$

Even with the spherical symmetry, in order to make contact with the experiment, one still needs to calculate a very large number of orbitals. For example, in our largest simulation performed so far, the angular quantum number $l$ was as high as 300 and the radial quantum number $k$ of the discrete states was as high as 18 . The dimensions of this nanoshell were $(16,19) \mathrm{nm}$ and, from an experimental point of view, this will be considered a very small nanoshell. The success of such large calculations clearly depends on how fast each iteration is performed.

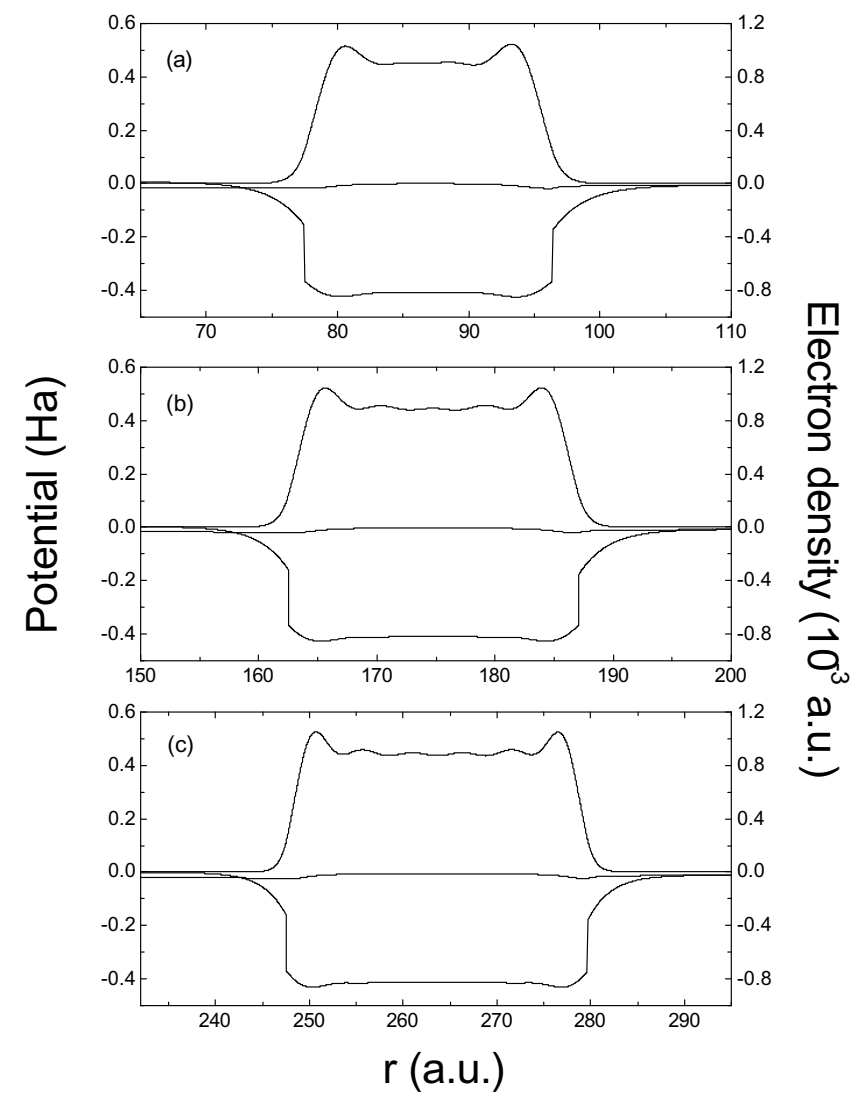

Figure 2. The electronic structure of three different nanoshells which were experimentally fabricated and characterized by optical measurements. In each panel, the curves represent, from bottom and up, the effective and Coulomb potential (left axis) and the electron density (right axis). The dimensions of the nanoshells are: (4.1,5.1) nm (panel a), (8.6,9.9) $\mathrm{nm}$ (panel b) and $(13.1,14.9) \mathrm{nm}$ (panel c). 
The radial Kohn-Sham orbitals and energies are calculated using the shooting method. This method consists of finding two, generally independent, solutions of the radial Kohn-Sham equation corresponding to an arbitrary energy. One solution is regular in the origin and the other is regular at infinity. ${ }^{9}$ These solutions are found by direct integration of the radial Kohn-Sham equation using the fourth order Runge-Kutta method. The eigenvalues correspond to those particular values of the energy where the two solution become linearly dependent, i.e. their Wronskian is zero. We notice that this approach provides the eigenvalues and the orbitals in the same time. To find all eigenvalues, one will have to sweep the energy from the bottom of the effective potential to zero and record the points where the Wronskian changes sign. If there were no relation between different eigenvalues this will represent a prohibitively time consuming step. Fortunately, for a shell geometry, the radial Schrodinger equations corresponding to consecutive angular quantum numbers $l$ have very close eigenvalues and $\varepsilon_{l+1, k}$ is always above $\varepsilon_{l, k}$. This means we can reduce the sweeping interval to small intervals around $\varepsilon_{l, k}, k=0,1,2, \ldots$, in order to find the eigenvalues corresponding to $l+1$. Thus, all the $l>0$ orbitals can be calculated very efficiently. This is the reason why the shell geometry allows calculations with such large number of particles. Suppose we keep the thickness of the shell $d$ constant and we increase the outer radius $b$ of the shell. In this case the maximum value of the radial quantum number $k$ remains constant while the number of particles increases approximately as $N=3 d b^{2} / r_{s}^{3}$. This means that, as the overall size of the shell is increased, only orbitals with higher angular quantum number appear and, as we argued above, they can be calculated very efficiently.

Another difficult problem we encountered in our large simulations is the convergence of the iterative process. It was already pointed out in the literature that the iterative methods in general have convergence problem for large many-body systems. ${ }^{17}$ Our studies on the thermodynamic limit of the Kohn-Sham and Hartree equations revealed that this convergence problem is due to the long range of the Coulomb interaction. ?,?, 20 The solution to this problem is to replace the Coulomb interaction with a screened Coulomb interaction in the Hartree potential:

$$
v_{H} \rightarrow \int \frac{e^{-|\vec{x}-\vec{y}| / \lambda}}{|\vec{x}-\vec{y}|}\left[n(\vec{y})-n_{0}\right] d \vec{y} .
$$

The iteration converges fast for a small screening lengths $\lambda$ and suppose the result is $n_{\lambda}$. The next step is to increase the screening length $\lambda$ by a finite amount and start again the iteration process with $n_{\lambda}$ as the initial input. This is repeated until the results stabilizes. In the present calculations, the screening length was increased by 2 a.u. and, for each $\lambda, 8$ iterations were needed to achieve the convergence. The first four decimals of the eigenvalues become independent of $\lambda$ for $\lambda \gtrsim 40$ a.u..

Experimentally, the metallic shells are grown over a solid dielectric, which so far was either $\mathrm{Au}_{2} \mathrm{~S}$ or silica, and they are usually suspended in solution during measurements. Also, the gold ion cores are highly polarizable due to the completely filled d-band. Consequently, the conduction electrons are embedded in a highly polarizable medium. The effect of these dielectric media can be included in the electronic structure calculations as follows. Let us consider a nonuniform, possibly frequency dependent dielectric,

$$
\varepsilon(r)=\left\{\begin{array}{l}
\varepsilon_{1}=\varepsilon_{C} \text { for } r<r_{1} \\
\varepsilon_{2}=\varepsilon_{J} \text { for } r_{1}<r<r_{2} \\
\varepsilon_{3}=\varepsilon_{E} \text { for } r_{2}<r,
\end{array}\right.
$$

where $r_{1}$ and $r_{2}$ represents the inner, respectively outer radius of the shell. This parameterization accounts for the presence of a dielectric core, polarizable jellium background (metal cores) and a dielectric embedding medium. The Hartree term,

$$
v_{H}(\vec{r})=\int \frac{n\left(\vec{r}^{\prime}\right)-n_{0}}{\left|\vec{r}-\vec{r}^{\prime}\right|} d \vec{r}^{\prime},
$$

which essentially represents the electrostatic potential generated by the conduction electrons and the positive ion cores, has to be modified such that it satisfies the usual boundary conditions at the interface between different dielectric media: ${ }^{21}$

$$
\tilde{v}_{H}(\vec{r})=\left\{\begin{array}{l}
v_{H}(\vec{r}) / \varepsilon_{C}+\Phi_{C} \text { for }|\vec{r}|<r_{1} \\
v_{H}(\vec{r}) / \varepsilon_{J}+\Phi_{J} \text { for } r_{1}<|\vec{r}|<r_{2} \\
v_{H}(\vec{r}) / \varepsilon_{E} \text { for } r_{2}<|\vec{r}|,
\end{array}\right.
$$


The constants are found from the requirement that the potential is continuous and they are given by:

$$
\left\{\begin{array}{l}
\Phi_{J}=\left(1 / \varepsilon_{E}-1 / \varepsilon_{J}\right) v_{H}\left(r_{2}\right) \\
\Phi_{C}=\Phi_{J}+\left(1 / \varepsilon_{J}-1 / \varepsilon_{C}\right) v_{H}\left(r_{1}\right) .
\end{array}\right.
$$

In Fig. 1, we show the effect of the dielectric media on the electronic structure of the nanoshell. The values for the dielectric constants were chosen as $\varepsilon_{C}=5, \varepsilon_{E}=2$ and $\varepsilon_{J}=8$ which are representative for realistic situations. The influence of $\varepsilon_{C}$ and $\varepsilon_{E}$ is relatively weak. The strongest influence on the electronic structure comes from $\varepsilon_{J}$ which strongly reduces the dipole fields near the surfaces. This leads to a lowering of the effective potential in the nanoshell. The reduced workfunction results in an increased electron spill out at the surfaces of the shell. The effect of the d-electrons is slightly reduced when the core and embedding medium are added. Although the dielectric effects on the electronic structure are visible, these variations alone have little influence on the optical response of the nanoparticles. ${ }^{10}$ This is in contrast to what have been observed experimentally, i.e. large shifts in the plasmon frequencies due to the presence of different dielectric media. As will be argued in the next section, the explanation is that the system responds differently to an external electric field in the presence of the dielectrics which screen the excitation.

Figure 2 illustrates the converged electronic structure of three nanoshells which were fabricated and characterized by optical measurements. The electronic structure shown in this figure will be later used to calculate the optical response of these nanoshells and make a direct comparison with the experiment. The dimensions of these nanoshells are $(4.1,5.1) \mathrm{nm},(8.6,9.9) \mathrm{nm}$ and $(13.1,14.9) \mathrm{nm}$. The largest nanoparticle contains about $2.5 \times 10^{5}$ conduction electrons. The nanoshells were suspended in an aqueous solution $\left(\varepsilon_{E}=1.78\right)$ and their core consisted of $\mathrm{Au}_{2} \mathrm{~S}\left(\varepsilon_{C}=5.4\right)$. The value of the jellium dielectric constant was fixed to $\varepsilon_{J}=8$, determined from a fit of the experimentally measured gold dielectric function with a Drude like expression.

The independent electron response function is calculated directly from the electronic structure. We are interested here only in the dipolar term $(l=1)$ of the response function, which is calculated using the following expansion: ${ }^{14}$

$$
\begin{array}{r}
\tilde{\Pi}_{l=1}^{0}\left(r, r^{\prime} ; \omega\right)=\sum_{\left|l_{1}-l_{2}\right|=1} \frac{l_{1}+l_{2}+1}{4 \pi} \sum_{k} f_{\beta}\left(\varepsilon_{k l_{1}}\right) u_{k l_{1}}(r) u_{k l_{1}}\left(r^{\prime}\right) \\
\times\left[G_{l_{2}}\left(r, r^{\prime} ; \varepsilon_{k l_{1}}+\omega+i \delta\right)+G_{l_{2}}\left(r^{\prime}, r ; \varepsilon_{k l_{1}}-\omega-i \delta\right)\right]
\end{array}
$$

where $G_{l}, l=0,1, \ldots$, represent the radial Green functions:

$$
\left(-\frac{1}{2} \frac{d^{2}}{d r^{2}}+\frac{l(l+1)}{2 r^{2}}+v_{e f f}(r)-E-i \delta\right) G_{l}\left(r, r^{\prime} ; E+i \delta\right)=-\delta\left(r-r^{\prime}\right),
$$

and $u_{k l}$ are the self-consistently calculated Kohn-Sham orbitals. The Green functions are calculated by direct integration of their specific equation. Eq. (11) provides the response function multiplied by $r^{2} r^{\prime 2}$ which is more convenient to use as it will follow from the next section.

\section{OPTICAL RESPONSE}

We will focus in this section on deriving the RPA equations in the presence of the dielectric medium defined by Eq. (7). The physical picture can be described as follow. When the whole system is placed in an external field, the conduction electrons will try to screen the excitation field. The dielectrics will polarize when placed in the external field, screening at their turn the excitation field. The conduction electrons will screen not only the original excitation but also the electric fields produced by the polarized dielectrics. To quantify these processes, we start from the TDLDA expression of the screening charge induced by an external electric field $E_{0} e^{-i \omega t} \vec{e}_{z}$,

$$
\delta n(\vec{r}, \omega)=\int d \overrightarrow{r^{\prime}} \Pi^{(0)}\left(\vec{r}, \vec{r}^{\prime} ; \omega\right)\left[\delta v_{x c}\left(\vec{r}^{\prime}, \omega\right)+\phi_{C}\left(\vec{r}^{\prime}, \omega\right)\right],
$$


where $\Pi^{(0)}$ represents the response function of the independent electrons. The quantity $\delta v_{x c}$ represents the variation of the exchange-correlation potential due to the screening charge and $\phi_{C}$ represents the Coulomb potential generated by the screening charges $\delta n$ and including the excitation field. In the quasistatic limit, which is generally accepted to be valid for particles smaller than $40 \mathrm{~nm},{ }^{22,23}$ the electrtatic potential $\phi_{C}$ must satisfy the Poisson equation with the appropriate boundary conditions:

$$
\left\{\begin{array}{l}
\varepsilon_{i} \vec{\nabla}^{2} \phi_{C}(\vec{r}, \omega)=-4 \pi \delta n(\vec{r}, \omega) \\
\left.\varepsilon_{i} \frac{\partial \phi_{C}}{\partial \vec{n}}\right|_{r=r_{i}^{-}}=\left.\varepsilon_{i+1} \frac{\partial \phi_{C}}{\partial \vec{n}}\right|_{r=r_{i}^{+}} \\
\phi_{C}(\vec{r}, \omega) \rightarrow-\vec{r} \vec{E}_{0} \text { for }|\vec{r}| \rightarrow \infty .
\end{array}\right.
$$

The solution can be written in the following form:

$$
\phi_{C}(\vec{r}, \omega)=\left(-r+\frac{4 \pi}{3} \int \frac{r_{<}}{r_{>}^{2} \varepsilon\left(r^{\prime}\right)} \delta n\left(r^{\prime}, \omega\right) r^{\prime 2} d r^{\prime}+\sum_{i=1,2} r_{i}^{2} \sigma^{(i)} v_{1}\left(r, r_{i}\right)\right) E_{0} \cos \theta .
$$

The surface charges $\sigma^{1,2}$ are found from the boundary condition as described in a previous application. ${ }^{12}$ After the solution is plugged back into Eq. (13) and using the notation $v_{1}\left(r, r^{\prime}\right)=r_{<} / r_{>}^{2}, v_{2}\left(r, r^{\prime}\right)=d v_{1} / d r$ and $\alpha(r, \omega)=r^{2} \delta n(r, \omega)$, the RPA equation becomes:

$$
\begin{aligned}
& \alpha(r, \omega)-\int d r^{\prime} \tilde{\Pi}_{1}^{(0)}\left(r, r^{\prime} ; \omega\right) r^{\prime-2} v_{x c}^{\prime}\left(n\left(r^{\prime}\right)\right) \alpha\left(r^{\prime}, \omega\right) \\
& -\frac{4 \pi}{3} \int d r^{\prime} \int d r^{\prime \prime} \tilde{\Pi}_{1}^{(0)}\left(r, r^{\prime} ; \omega\right) \frac{v_{1}\left(r^{\prime}, r^{\prime \prime}\right)}{\varepsilon\left(r^{\prime \prime}\right)} \alpha\left(r^{\prime \prime}, \omega\right) \\
& -\frac{4 \pi}{3} \sum_{i=1,2} X^{(i)}(r, \omega) \int \frac{v_{2}\left(r_{i}, r^{\prime}\right)}{\varepsilon\left(r^{\prime}\right)} \alpha\left(r^{\prime}, \omega\right) d r^{\prime} \\
= & \int d r^{\prime} \tilde{\Pi}_{1}^{(0)}\left(r, r^{\prime} ; \omega\right) \phi_{C}^{0}\left(r^{\prime}\right),
\end{aligned}
$$

where

$$
\begin{aligned}
& X^{(1)}(r, \omega)=-\frac{1}{\xi_{\varepsilon}} \frac{\varepsilon_{C}-\varepsilon_{J}}{\varepsilon_{C}+2 \varepsilon_{J}} X_{1}(r, \omega)-\frac{\xi_{\varepsilon}-1}{\xi_{\varepsilon}} X_{2}(r, \omega) \\
& X^{(2)}(r, \omega)=\frac{\xi_{\varepsilon}-1}{2 \xi_{\varepsilon}}\left(\frac{r_{2}}{r_{1}}\right)^{3} X_{1}(r, \omega)+\frac{1}{\xi_{\varepsilon}} \frac{\varepsilon_{E}-\varepsilon_{J}}{\varepsilon_{J}+2 \varepsilon_{E}} X_{2}(r, \omega) .
\end{aligned}
$$

and

$$
X_{i}(r, \omega)=r_{i}^{2} \int d r^{\prime} \tilde{\Pi}_{1}^{(0)}\left(r, r^{\prime} ; \omega\right) v_{1}\left(r^{\prime}, r_{i}\right) .
$$

In these equations, $\phi_{C}^{0}$ represents the unscreened response of the dielectrics to the electric field:

$$
\phi_{C}^{0}(\vec{r})=\left(-r+\sum_{i=1,2} r_{i}^{2} \sigma_{0}^{(i)} v_{1}\left(r, r_{i}\right)\right) E_{0} \cos \theta,
$$

where

$$
\begin{aligned}
\sigma_{0}^{(1)} & =\frac{1}{\xi_{\varepsilon}} \frac{\varepsilon_{C}-\varepsilon_{J}}{\varepsilon_{C}+2 \varepsilon_{J}} \frac{3 \varepsilon_{E}}{\varepsilon_{J}+2 \varepsilon_{E}} \\
\sigma_{0}^{(2)} & =1-\frac{1}{\xi_{\varepsilon}} \frac{3 \varepsilon_{E}}{\varepsilon_{J}+2 \varepsilon_{E}}
\end{aligned}
$$

are the classical polarization charges and $\xi_{\varepsilon}$ is just a dielectric factor,

$$
\xi_{\varepsilon}=1-2 \frac{\varepsilon_{C}-\varepsilon_{J}}{\varepsilon_{C}+2 \varepsilon_{J}} \frac{\varepsilon_{E}-\varepsilon_{J}}{\varepsilon_{J}+2 \varepsilon_{E}}\left(\frac{r_{1}}{r_{2}}\right)^{3} .
$$




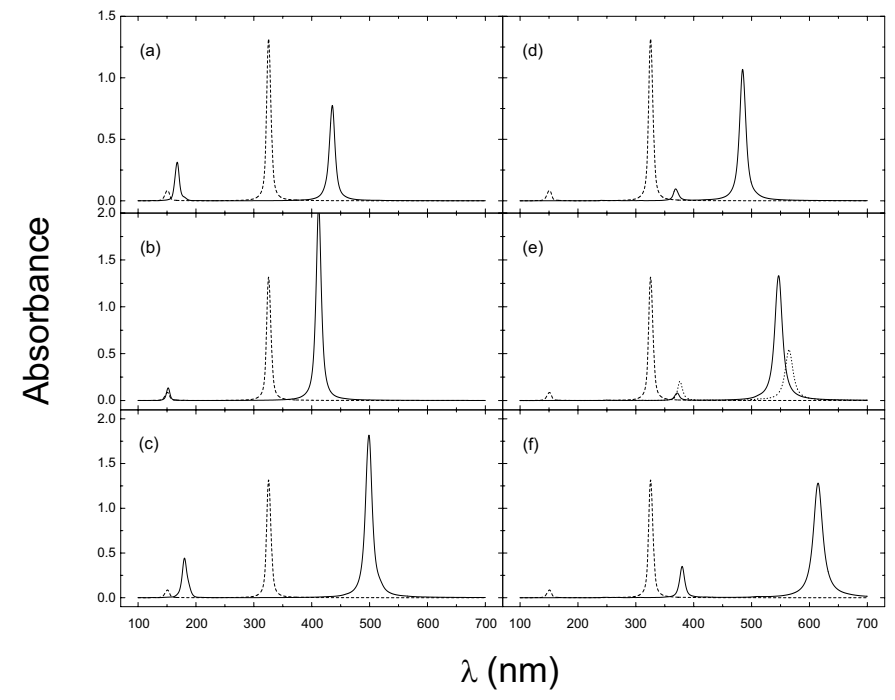

Figure 3. The effect of different dielectrics on the optical absorption of the gold nanoshell shown in Fig. 1. Different panels refer to the following situations: a) $\varepsilon_{C}=5, \varepsilon_{E}=1$ and $\varepsilon_{J}=1$; b) $\varepsilon_{C}=1$, $\varepsilon_{E}=2$ and $\varepsilon_{J}=1$; c) $\varepsilon_{C}=5$, $\varepsilon_{E}=2$ and $\varepsilon_{J}=1$; d) $\varepsilon_{C}=1, \varepsilon_{E}=1$ and $\varepsilon_{J}=8$; e) $\varepsilon_{C}=1, \varepsilon_{E}=2$ and $\varepsilon_{J}=8$ (solid) and $\varepsilon_{C}=5, \varepsilon_{E}=1$ and $\varepsilon_{J}=8$ (dotted); f) $\varepsilon_{C}=5, \varepsilon_{E}=2$ and $\varepsilon_{J}=8$. For comparison, in all panels we have included the absorbance of the gold nanoshell when no core or embedding medium is present and $\varepsilon_{J}=1$ (dashed line).

The integrals in the above equations are discretized and the integral equations are transformed in algebraic equations which are solved by conventional methods. ${ }^{9,} 10$ The photo absorption cross section of the nanoshell is related to its polarizability $\alpha(\omega)$ through

$$
\sigma_{a b s}(\omega)=\sqrt{\varepsilon_{E}} \frac{\omega}{c} \operatorname{Im}[\alpha(\omega)],
$$

where SI units were used. The frequency dependent polarizability is calculated from

$$
\alpha(\omega)=\frac{4 \pi}{3} \int d r r^{3} \alpha(r, \omega) .
$$

where $\alpha(r, \omega)$ is the local polarizability derived from the RPA equation Eq. (17). The absorbance is defined by the ratio between the optical absorption cross section and $\pi r_{2}^{2}$.

In Fig. 3, we show how the photoabsorption cross section of a $(70,100)$ a.u. nanoshell is influenced by the presence of dielectric media. For each nanoshell, the photoabsorption spectrum is dominated by two peaks, corresponding to the symmetric and antisymmetric coupling between charges on the inner and outer surfaces of the shell. The nanoshell plasmons can be viewed as bonding and antibonding superpositions of a cavity plasmon located on the inner surface of the shell and a sphere surface plasmon on the outer surface of the shell. In the presence of dielectric backgrounds, the energy of the cavity plasmon is $\omega_{C}=\omega_{B} \sqrt{\frac{2}{\varepsilon_{C}+2 \varepsilon_{J}}}$ and the energy of the sphere plasmon is $\omega_{S}=\omega_{B} \sqrt{\frac{1}{2 \varepsilon_{E}+\varepsilon_{J}}}$ The low energy (long wavelength) symmetric nanoshell plasmon $\omega_{-}$ has a larger admixture of the sphere plasmon and the high energy (low wavelength) antisymmetric mode $\omega_{+}$ is primarily composed of the the cavity plasmon. The changes in the optical spectra in Fig. 3 caused by the different dielectric media can be simply understood from how the bare cavity and sphere plasmon shifts in the presence of dielectrics. The $\omega_{+}$plasmon with its large cavity plasmon content is more strongly influenced by a 


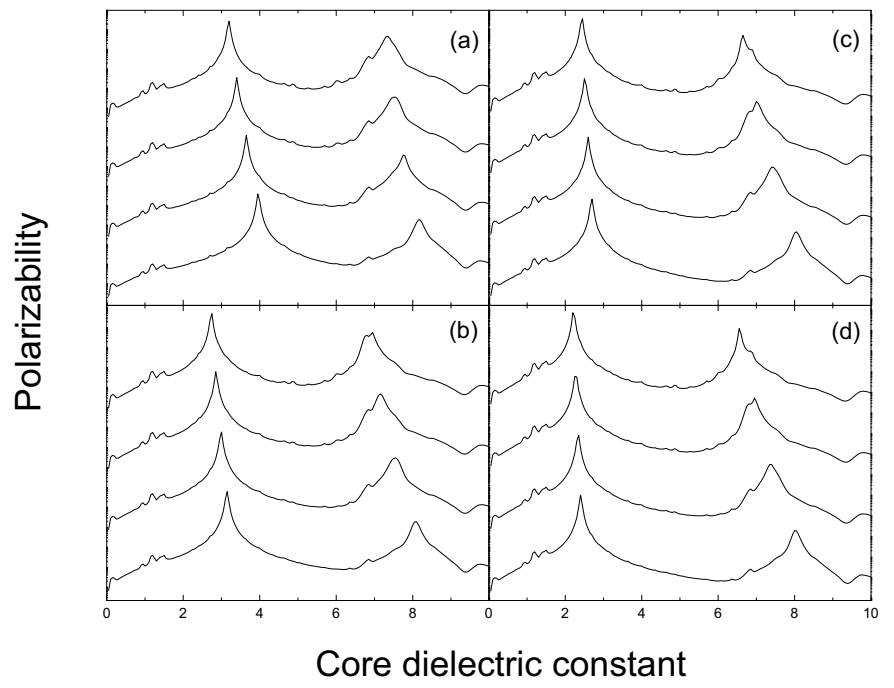

Figure 4. The optical response of a $(60,90)$ a.u. gold nanoshell when the dielectric constant of the core and embedding medium are varied over a range of 1 to 4 . The dielectric constant of the jellium shell $\varepsilon_{J}=1$. A logarithmic scale is used on the $\mathrm{y}=$ axis. Each panel, from the bottom curve and up, represents the optical response corresponding to a dielectric core of $1,2,3$ and 4. Different panels corresponds to $\varepsilon_{E}=1$ ( panel a), $\varepsilon_{E}=2$ (panel b), $\varepsilon_{E}=3$ (panel c) and $\varepsilon_{E}=4$ (panel d).

dielectric core $\varepsilon_{C}$ than by the embedding medium. The $\omega_{-}$plasmon is more strongly influenced by the dielectric embedding medium $\varepsilon_{E}$ than by the dielectric core. The presence of a dielectric jellium background $\varepsilon_{J}$ has a stronger influence on $\omega_{+}$simply because the cavity plasmon depend more strongly on $\varepsilon_{J}$ than the surface sphere plasmon.

In Fig. 4 we calculate the optical absorption spectra of a $(60,90)$ a.u. for a broad range of values of the core and embedding medium dielectric constant. The optical absorption is plotted in a logarithmic scale. The figure show that in addition to the two collective resonances $\omega_{+}$and $\omega_{-}$there is a background contribution to the optical absorption. This background absorption is caused by discrete single particle excitations. It can be seen that as the dielectric constant of the core and embedding medium is changed, only the collective plasmon resonances are influenced. The discrete single particle excitations remain essentially unchanged because the presence of dielectric media only weakly influences the electronic structure of the system. A calculation of the polarizability only including the changes in the electronic structure caused by the dielectrics has almost no effect on the optical properties of the nanoshell. ${ }^{10}$ The large effect of a dielectric core or embedding medium on the plasmon resonances is due to the polarization of the dielectrics and their contribution to $\phi_{C}$ in Eq. (13).

The frequency of the plasmon resonances extracted from the optical absorption spectra shown in figure Fig. 4 are in excellent agreement with the predictions of the classical Mie theory as it is illustrated in Fig. 5. In the classical calculations, the metallic phase is modeled by a pure Drude dielectric function corresponding to the same $r_{s}=3$ as used in the TDLDA calculations.

The fundamental property of the metallic nanoshells is their tunable plasmon resonances, which was demonstrated in a series of experiments where monodisperse nanoshells of different shell thickness and core sizes have been fabricated and characterized with optical measurements. ${ }^{24}$ In the absence of any dielectrics, the classical 


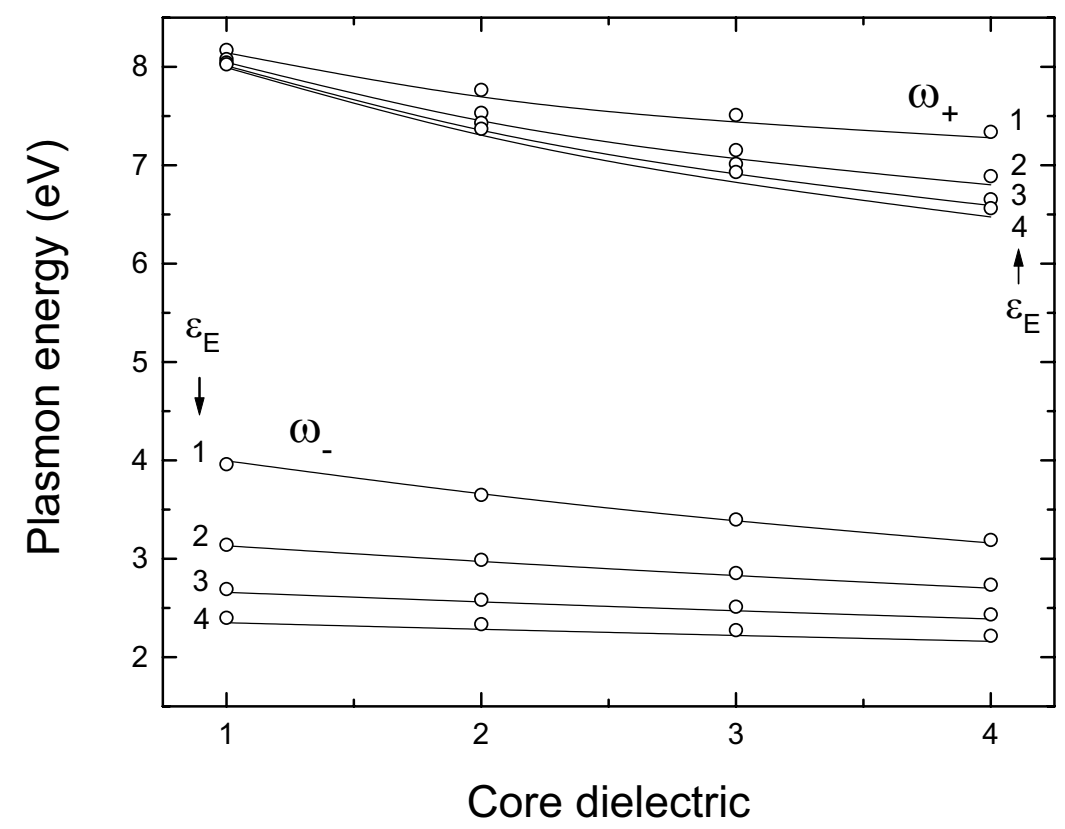

Figure 5. The TDLDA calculated plasmon energies (circles) compared with the prediction of the classical Mie theory (solid lines) for different core dielectrics and embedding media.

Mie theory predicts the following expression for the plasmon energies:

$$
\omega_{l \pm}^{2}=\frac{\omega_{B}^{2}}{2}\left[1 \pm \frac{1}{2 l+1} \sqrt{1+4 l(l+1) x^{2 l+1}}\right]
$$

which depends only on the aspect ratio of the nanoshell $x=r_{1} / r_{2}$. The same expression can be derived using semi-classical approaches. ${ }^{25}$ We show in the following that our self-consistent calculations lead to the same aspect ratio dependence of the plasmon energies. For this, we fix the dielectric constants to 1 and we apply the TDLDA formalism to nine nanoshells of the same thickness (17 a.u.) but with different overall diameters, ranging from 85 to 680 a.u.. The aspect ratio of the nanoshell is varied in this way over a broad range of values starting from 0.6 and up to 0.95 . From the polarizability curves we extract the wavelengths of the collective modes which are shown in Fig. 6 by open circles. The agreement between the TDLDA calculated plasmon wavelengths and Eq. (24) is almost perfect over the entire range of aspect ratios. The lower energy plasmon becomes strongly dependent on the aspect ratio for thin shells when the interaction between the cavity and sphere plasmon becomes very strong. This is the regime where the nanoshells become very useful in a variety of applications.

\section{COMPARISON WITH EXPERIMENTAL DATA}

In this section we apply the formalism presented in the last section and calculate the optical response of the three nanoshells presented in Fig. 2. The procedure for calculating an effective jellium background dielectric function consists of the following. The conduction electrons are assumed to contribute to the dielectric function of the metal through a Drude term:

$$
\varepsilon_{D}(\omega)=1-\omega_{B}^{2} /\left(\omega^{2}+i \gamma \omega\right) .
$$




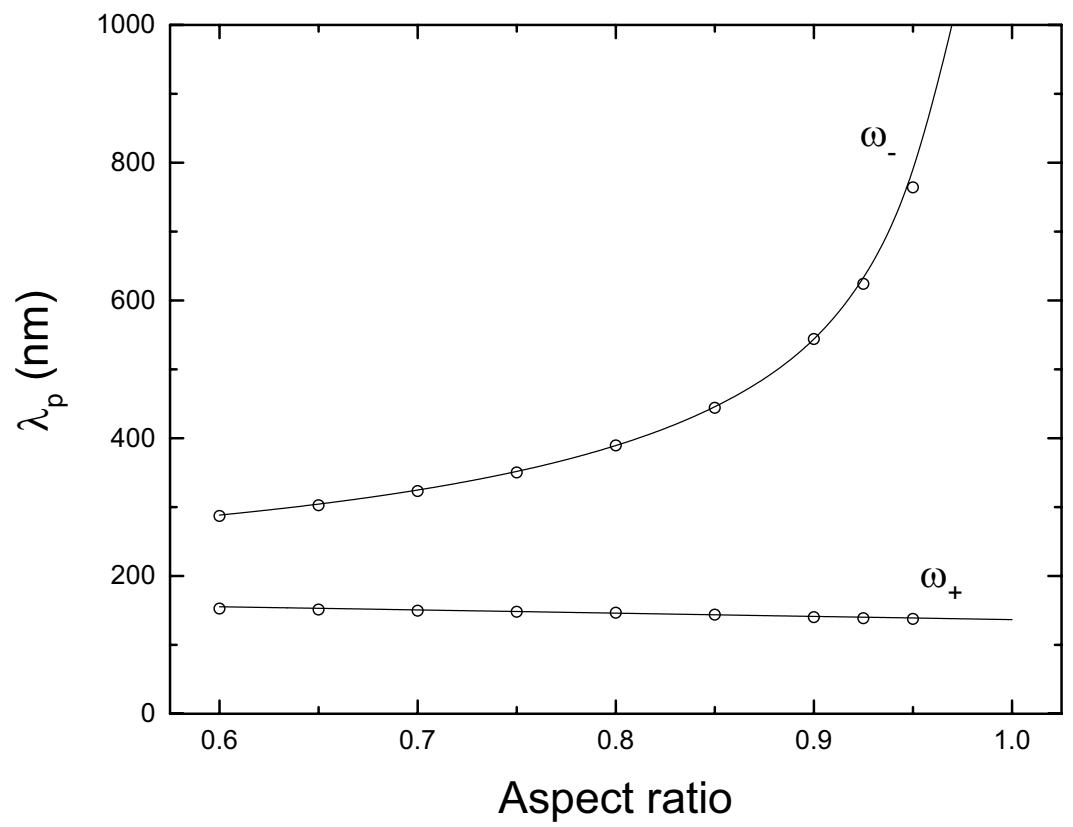

Figure 6. The TDLDA calculated plasmon wavelengths (circles) compared with the prediction of the classical Mie theory Eq. (24) (solid lines) for nanoshells of different aspect ratios. The TDLDA has been applied to a set of nine nanoshells of the same thickness (17 a.u.) and with overall diameters of 85, 97.2, 117.4, 136, 170, 226.6, 340, 453 and 680 a.u..

This assumption is supported by the excellent agreement between the TDLDA calculations and the classical Mie calculations when the metallic phase is modeled by a Drude dielectric function. The width $\gamma$ should be the same as the artificial width that will be used in the TDLDA calculations. ${ }^{26}$ The experimentally measured bulk dielectric function of gold, ${ }^{27} \varepsilon^{e x p}(\omega)$ can then be decomposed into: ${ }^{26,28}$

$$
\varepsilon^{e x p}(\omega)=\varepsilon_{J}(\omega)+\varepsilon_{D}(\omega)-1,
$$

which provides a simple way of determining the jellium dielectric function $\varepsilon_{J}(\omega)$. The RPA equation Eq. (17) have been solved with the frequency dependent jellium dielectric function obtained by this procedure and the results are shown in Fig. 7 by solid lines. We also show a calculation using a frequency independent $\varepsilon_{J}=8$ obtained from a best fit of $\varepsilon_{J}(\omega)$ to a constant. The agreement with the experimentally measured optical absorption shown by circles is excellent for all three nanoshells. The experimental spectra show an additional peak for all three nanoshells which is located at the same wavelength of about $520 \mathrm{~nm}$. This peak is generated by solid gold colloids also present in the solution during the experiment. The peak at larger wavelengths is the $\omega_{-}$resonant mode and its position is different for the three nanoshells, which have different aspect ratios. The $\omega_{-}$mode in the TDLDA calculations is slightly red shifted relative to the experiment.

In these experiments the nanoshells are not perfectly monodisperse and this is due to slight variations in their geometry. The size distribution was evaluated to a Gaussian of a standard deviation of $11 \%$, which can explain the difference in the widths of the plasmon resonances. To support this argument, we have calculated the optical response of nine nanoshells of sizes uniformly distributed in between $\pm 11 \%$ of the standard size. The results are shown in Fig. 8. The optical absorption curves have been multiplied by the corresponding Gaussian weight. As one can see, a size distribution of $11 \%$ introduces a large broadening of the plasmons. This is mainly due 


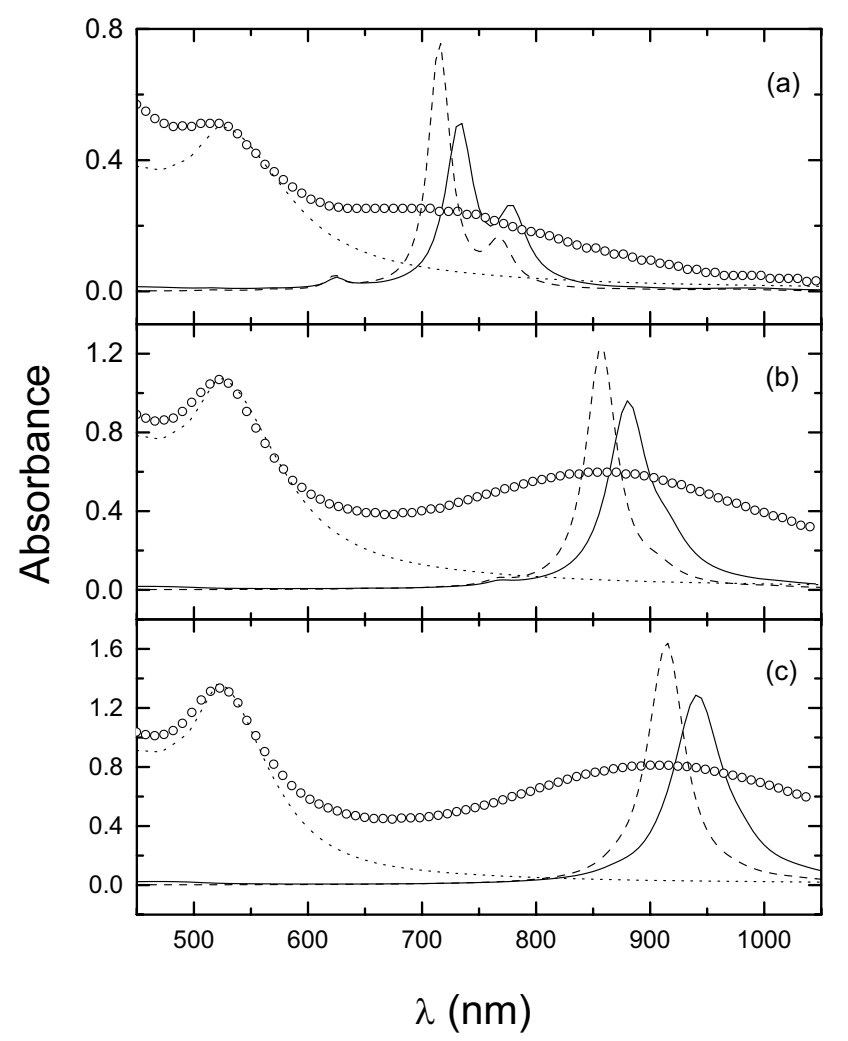

Figure 7. The optical response of three gold nanoshells with $\mathrm{Au}_{2} S$ core of geometries $(4.1,5.1) \mathrm{nm}$ (panel a), (8.6,9.9) nm (panel b) and $(13.1,14.9) \mathrm{nm}$ (panel c). The solid curves represent the TDLDA calculation when the full frequency dependent dielectric constant of gold is used. The dashed lines represent the TDLDA calculation when a Drude dielectric function with $\varepsilon_{J}=8$ is used. The circles represent the experimentally measured optical response. The dotted lines represents the optical absorption of solid gold particles also present in the solution during the experiment.

to the fact that the thickness of the nanoshells is very small compared with the overall size and, in this regime, the plasmon modes are very sensitive to small variations of the geometry as it was illustrated in Fig. 6. Also, the size distribution induces a blue shift because the height of the plasmon peaks increases with the size of the nanoshells. The figure clearly shows that the variation of the plasmon energies caused by the inhomogeneous size distribution can account for the experimental broadening of the plasmon resonance.

\section{CONCLUSIONS}

The optical properties of large nanoshells can be calculated using the TDLDA method. The results show that the plasmon energies are sensitive to geometry and can depend strongly on the presence of dielectric backgrounds such as a dielectric core, jellium background and embedding medium. The calculated energies of the plasmon resonances are in excellent agreement with classical Mie scattering. A comparison with experimental data show very good agreement between the theoretical and experimental absorption spectra. 


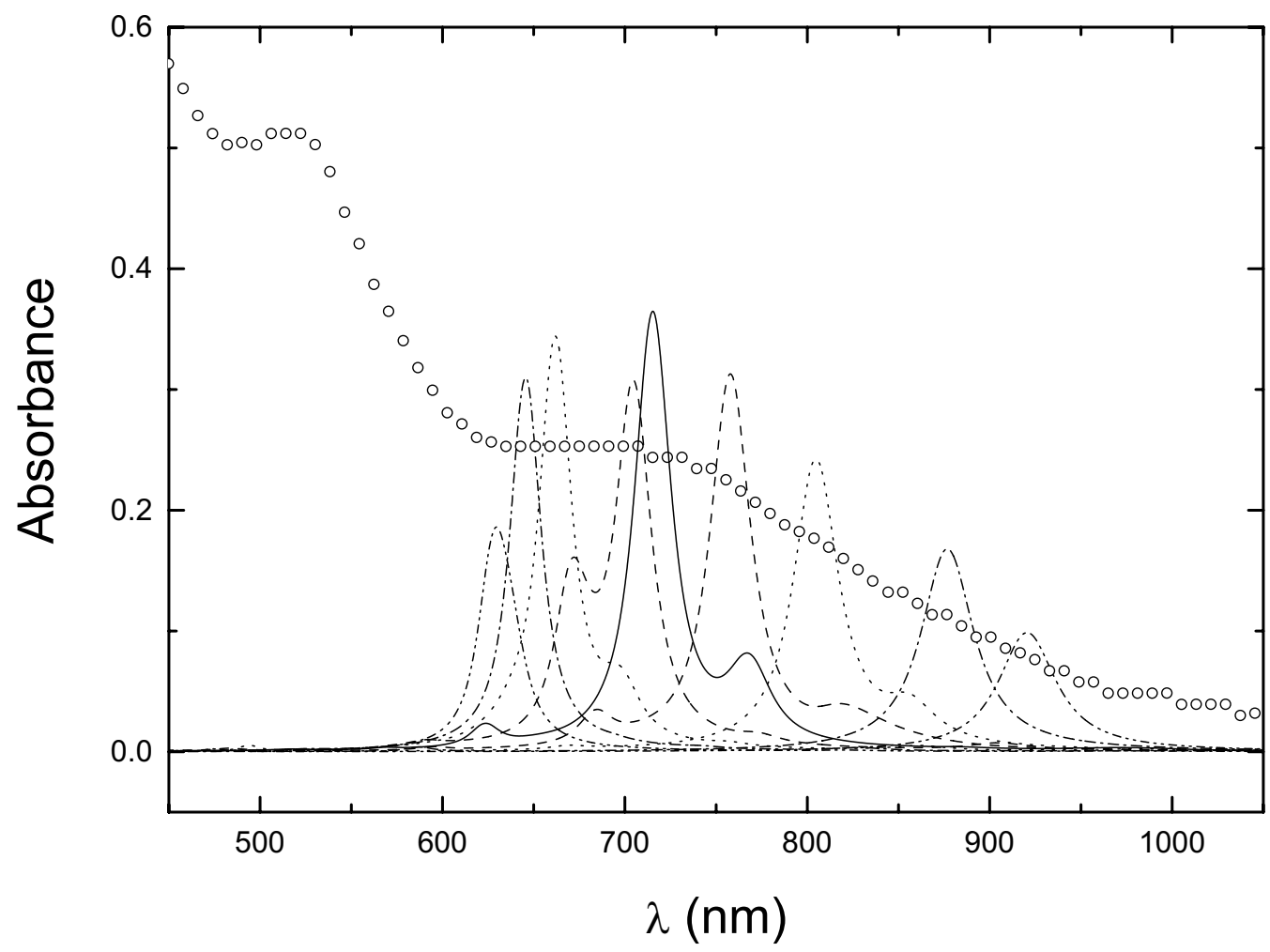

Figure 8. The effect of a size distribution on the optical absorption of a solution of gold nanoshells. The figure illustrates the TDLDA optical absorption of different nanoshells of slightly different geometries, $\delta= \pm 2.75 \%$ (dashed), $\delta= \pm 5.5 \%$ (dotted), $\delta= \pm 8.25 \%$ (dashed-dotted) and $\delta= \pm 11 \%$ (dashed-dotted-dotted) relative to the geometry (4.1,5.1) nm (solid). Each curve has been multiplied by a Gaussian factor $\alpha e^{-(\delta / \sigma)^{2}}$, where $\sigma=11 \%$ as predicted from experiment and $\alpha$ was chosen equal to 0.5 . The circles represent the experimentally measured optical absorption.

\section{ACKNOWLEDGMENTS}

This work was supported by the Robert A. Welch foundation under grant C-1222, by the Texas Advanced Technology Program, and by the Multi-University Research Initiative of the Army Research Office.

\section{REFERENCES}

1. M. Brongersma, "Nanoshells: gifts in a gold wrapper," Nat. Mater. 2, p. 296, 2003.

2. R. D. Averitt, D. Sarkar, and N. J. Halas, "Plasmon resonance shifts of au coated $\mathrm{Au}_{2} \mathrm{~S}$ nanoshells: Insight into multicomponent nanoparticle growth," Phys. Rev. Lett. 78, pp. 4217-4220, 1997.

3. G. D. Hale, J. B. Jackson, O. E. Shmakova, T. R. Lee, and N. J. Halas, "Enhancing the active lifetime of luminescent semiconducting polymers via doping with metal nanoshells," Appl. Phys. Lett. 78, pp. 15021504, 2001.

4. S. Sershen, S. L. Westcott, J. L. West, and N. J. Halas, "An opto-mechanical nanoshell-polymer composite," Applied Physics B73, pp. 379-381, 2001. 
5. S. Sershen, S. L. Westcott, N. J. Halas, and J. L. West, "Temperature-sensitive polymer-nanoshell composites for photothermally modulated drug delivery," J. Biomed. Mat. Res. 51, pp. 293-298, 2000.

6. Y. Sun and Y. Xia, "Increased sensitivity of surface plasmon resonace of gold nanoshells compared to that of gold solid colloids in response to environmental changes," Anal. Chem. 74, pp. 5297-5305, 2002.

7. J. B. Jackson, S. L. Westcott, L. R. Hirsch, J. L. West, and N. J. Halas, "Controlling the surface enhanced raman effect via the nanoshell geometry," Appl. Phys. Lett. 82, pp. 257-259, 2003.

8. E. Prodan and P. Nordlander, "Exchange and correlation effects in small metallic nanoshells," Chem. Phys. Lett. 349, pp. 153-160, 2001.

9. E. Prodan and P. Nordlander, "Electronic structure and polarizability of metallic nanoshells," Chem. Phys. Lett. 352, pp. 140-146, 2002.

10. E. Prodan, A. Lee, and P. Nordlander, "The effect of a dielectric core and embedding medium on the polarizability of metallic nanoshells," Chem. Phys. Lett. 360, pp. 325-332, 2002.

11. P. Nordlander and E. Prodan, "Optical properties of metallic nanoshells," Proc. SPIE 4810, pp. 91-98, 2002.

12. E. Prodan, P. Nordlander, and N. J. Halas, "Effects of dielectric screening on the optical properties of metallic nanoshells," Chem. Phys. Lett. 368, pp. 94-101, 2003.

13. E. Prodan and P. Nordlander, "Structural tunability of the plasmon resonances in metallic nanoshells," Nano Letters 3, pp. 543-547, 2003.

14. A. Zangwill and P. Soven, "Density-functional approach to local-field effects in finite systems: Photoabsorption in the rare gases," Phys. Rev. A21, pp. 1561-1572, 1980.

15. W. de Heer, "The physics of simple metal clusters: experimental aspects and simple models," Rev. Mod. Phys. 65, pp. 611-676, 1993.

16. M. Brack, "The physics of simple metal clusters: self-consistent jellium model and semiclassical approaches," Rev. Mod. Phys. 65, pp. 677-732, 1993.

17. G. Bertsch, "An RPA program for jellium spheres," Computer Physics Communications 60 (2), pp. 247-255, 1990.

18. E. Prodan and P. Nordlander, "Hartree approximation I: The fixed point approach," J. Math. Phys. 42, pp. 3390-3406, 2001.

19. E. Prodan and P. Nordlander, "Hartree approximation II: The thermodynamic limit," J. Math. Phys. 42, pp. 3407-3423, 2001.

20. E. Prodan and P. Nordlander, "On the Kohn-Sham equations with periodic background potentials," J. Stat. Phys. 111, pp. 967-992, 2003.

21. A. Rubio and L. Serra, "Dielectric screening effects on the photo absorption cross section of embedded metallic clusters," Phys. Rev. B48, pp. 18222-18229, 1993.

22. U. Kreibig and M. Vollmer, Optical properties of metal clusters, Springer, New York, 1995.

23. C. Sonnichsen, T. Franzl, T. Wilk, and G. von Plessen, "Plasmon resonances in large noble metal clusters," New J. Phys. 4, p. 93, 2002.

24. S. J. Oldenburg, J. B. Jackson, S. L. Westcott, and N. J. Halas, "Infrared extinction properties of gold nanoshells," Appl. Phys. Lett. 75, pp. 2897-2899, 1999.

25. G. Mukhopadhyay and S. Lundqvist, "Density oscillations and density response in systems with nonuniform electron density," Il Nuovo Cim. 27B, pp. 1-17, 1975.

26. J. Lerme, B. Palpant, E. Cottancin, M. Pellarin, B. Prevel, J. L. Vialle, and M. Broyer, "Quantum extension of Mie's theory in the dipolar approximation," Phys. Rev. B60, pp. 16151-16156, 1999.

27. P. Johnson and R. Christy, "Optical constants of the noble metals," Phys. Rev. B6, p. 4370, 1972.

28. T. Shahbazyan, I. Perakis, and J. Bigot, "Size-dependent surface plasmon dynamics in metal nano particles," Phys. Rev. Lett. 81, pp. 3120-3123, 1998. 